\title{
An Adult Case of Chronic Active Epstein-Barr Virus Infection with Interstitial Pneumonitis
}

\author{
Eun-Jeong Joo', Young Eun Ha', Dong Sik Jung ${ }^{2}$, Hae Suk Cheong ${ }^{3}$, Yu Mi Wi', Jae-Hoon Song', \\ and Kyong Ran Peck ${ }^{1}$
}

${ }^{1}$ Division of Infectious Diseases, Samsung Medical Center, Sungkyunkwan University School of Medicine, Seoul; ${ }^{2}$ Division of Infectious Diseases, Dong-A University Hospital, Busan; ${ }^{3}$ Division of Infectious Diseases, Konkuk University Medical Center, Seoul; ${ }^{4}$ Division of Infectious Diseases, Samsung Changwon Hospital, Sungkyunkwan University School of Medicine, Changwon, Korea

Chronic active Epstein-Barr virus (CAEBV) infection is characterized by persistent infectious mononucleosis-like symptoms, an unusual pattern of Epstein-Barr virus (EBV) antibodies, detection of the EBV genome in affected tissues or peripheral blood, and chronic illness that cannot be attributed to any other known disease. This is the first reported Korean case of an immunocompetent adult with CAEBV-associated interstitial pneumonitis. A 28-year-old female was admitted with a fever that persisted for 3 weeks. She had multiple lymphadenopathy, hepatosplenomegaly, pancytopenia, and elevated serum aminotransferase levels. Serology for antibodies was positive and chest computed tomography showed diffuse ground glass opacities in both lungs. Histopathology of the lung tissue showed lymphocyte infiltration, and EBV DNA was detected in those lymphocytes using in situ hybridization with an EBV-encoded RNA probe. After 1 month of hospitalization, she improved without specific treatment.

Keywords: Epstein-Barr virus infection; Immunocompetence; Lung diseases; Interstitial pneumonitis

\section{INTRODUCTION}

Epstein-Barr virus (EBV) is a ubiquitous virus that can cause both acute and chronic active infections. Primary EBV infection is usually asymptomatic, but it can cause active symptomatic infection, including infectious mononucleosis, which resolves spontaneously after EBV-specific immunity develops [1]. EBV usually remains latent following the primary infection, although in some patients it progresses to chronic active infection characterized by a persistent infectious mononucleosis-like syndrome, which may include fever, persistent hepatitis, extensive lymph- adenopathy, hepatosplenomegaly, pancytopenia, high viral loads in peripheral blood, and an unusual pattern of EBVrelated antibodies [2]. Recently, Okano et al. [3] reviewed patients with persistent infectious mononucleosis-like syndrome and proposed diagnostic criteria for chronic active EBV (CAEBV) infection.

Chronic active EBV may result in life-threatening complications, such as hemophagocytic syndrome, disseminated intravascular coagulopathy, hepatic failure, coronary artery aneurysm, central nervous system involvement, myocarditis, lymphoma, and hematologic malignancies [2]. Rarely, interstitial pneumonitis occurs as a serious com-

Received : May 13, 2008

Revised : June 4, 2008

Accepted: July 17, 2008

Correspondence to Kyong Ran Peck, M.D.

Division of Infectious Diseases, Samsung Medical Center, Sungkyunkwan University School of Medicine, 50 Irwon-dong, Gangnam-gu, Seoul 135-710, Korea

Tel: 82-2-3410-0329, Fax: 82-2-3410-0041, E-mail: krpeck@skku.edu 
plication in CAEBV patients; here, we report the first such case in Korea. In this case, an immunocompetent adult had a clinical syndrome of CAEBV and interstitial pneumonitis associated with the infiltration of EBV-infected T lymphocytes into the lungs, which implies CAEBV-associated interstitial pneumonitis.

\section{CASE REPORT}

A 28-year-old female was admitted with a persistent fever for 3 weeks and flu-like symptoms. On admission, her temperature was $38.6^{\circ} \mathrm{C}$, blood pressure was $130 / 70$ $\mathrm{mmHg}$, and respiration rate was $30 / \mathrm{min}$. Her inguinal lymph nodes were palpable, about $0.5 \mathrm{~cm}$ in diameter, tender, and fixed. She had decreased breath sounds in both lower lung fields and hepatosplenomegaly. Her hemoglobin was $13.2 \mathrm{~g} / \mathrm{dL}$, platelet count $56,000 / \mu \mathrm{L}$, and leukocyte count 2,770 units (segmented neutrophils $48.7 \%$, lymphocytes $43 \%)$. Her serum bilirubin was 1.3 $\mathrm{mg} / \mathrm{dL}$, aspartate transaminase/alanine aminotransferase 610/697 U/L, serum protein $6.3 \mathrm{~g} / \mathrm{dL}$, serum albumin $3.5 \mathrm{~g} / \mathrm{dL}$, and lactate dehydrogenase $2,327 \mathrm{ng} / \mathrm{mL}$. Blood, urine, and pleural fluid cultures did not grow any bacteria or fungi. The pleural fluid was a transudate. The serologic tests showed EBV viral capsid antigen (VCA)-IgG (+), EBV VCA-IgM (-), EBV- early antigen (EA) (+), EBV nuclear antigen (EBNA) (+), anti-HBs Ab (+), and anti-HCV Ab $(-)$. The EBV-DNA copy number in whole blood, as measured by real-time polymerase chain reaction (RT-PCR), was 946.1 copies $/ 5 \mu \mathrm{L}$. The chest radiograph showed diffuse ground glass opacities in both lower lung fields with bilateral pleural effusions (Fig. 1). Computed tomography of the abdomen and chest revealed hepatosplenomegaly and diffuse ground glass opacities and interlobular septal thickening of the lungs (Fig. 2). Flexible bronchoscopy showed normal bronchial anatomy without inflammation of the airway mucosa. Histopathology of inguinal lymph node tissue showed reactive hyperplasia and was negative for EBV DNA. A normocellular marrow with three-lineage hematopoiesis and few small to medium-sized aggregated $\mathrm{CD}^{+} \mathrm{T}$ lymphocytes were observed in a bone marrow aspirate. A conventional PCR technique with an EBVencoded small RNA (EBER) probe detected EBV DNA in those lymphocytes.

Six days after admission, the patient was transferred to the intensive care unit because of impending respiratory

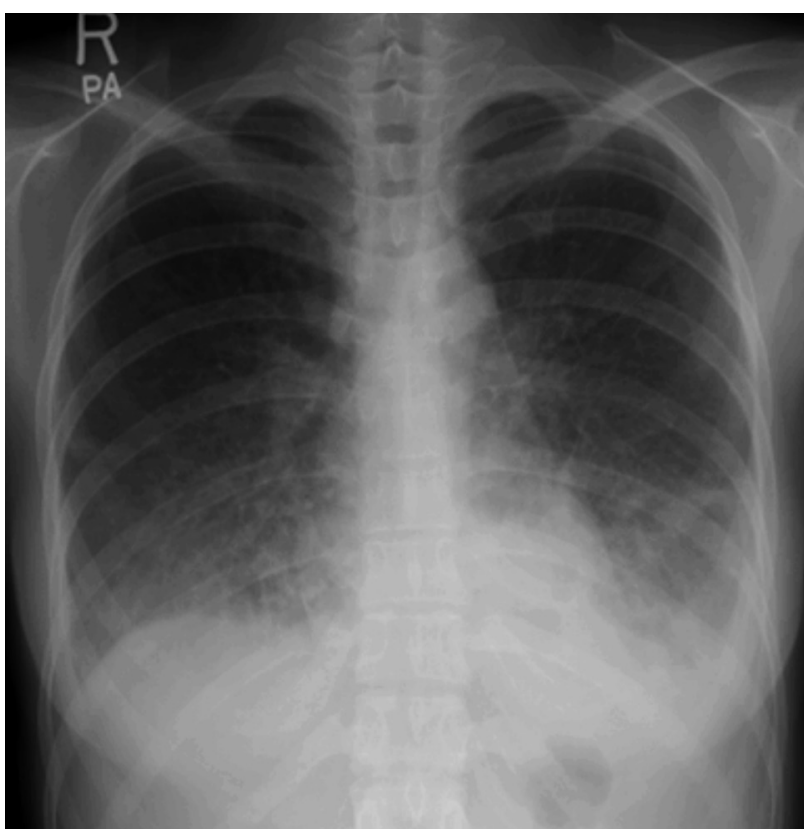

Figure 1. Diffuse ground glass opacity in both lower lung fields with bilateral pleural effusions.

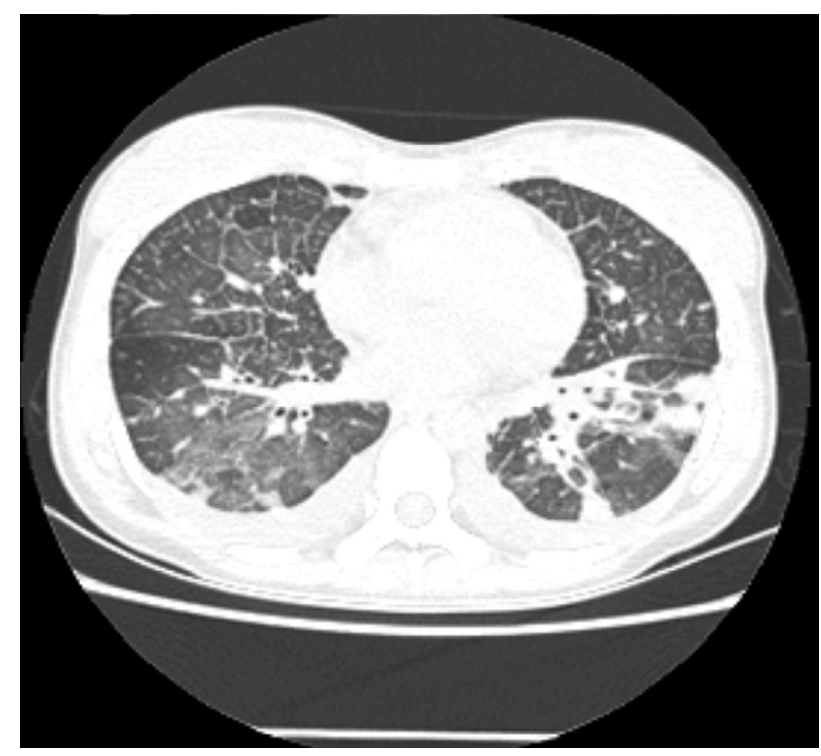

Figure 2. Diffuse ground glass opacity and interlobular septal thickening of the lungs.

failure. At this point, a lung biopsy was performed with video-assisted thoracoscopic surgery. Histopathologically, the lung tissue showed infiltration of the alveolar septum and peribronchial interstitium by small to large lymphocytes (Fig. 3). The infiltrate was composed predominantly of $\mathrm{CD}_{3}{ }^{+}$and $\mathrm{CD} 56-\mathrm{T}$ lymphocytes on immunohistochemistry (Fig. 4). EBV DNA was detected by in situ hybridization using an EBER probe on the lymphocytes (Fig. 5) and the $T C R \gamma$ gene arrangement showed a polyclonal pattern. 


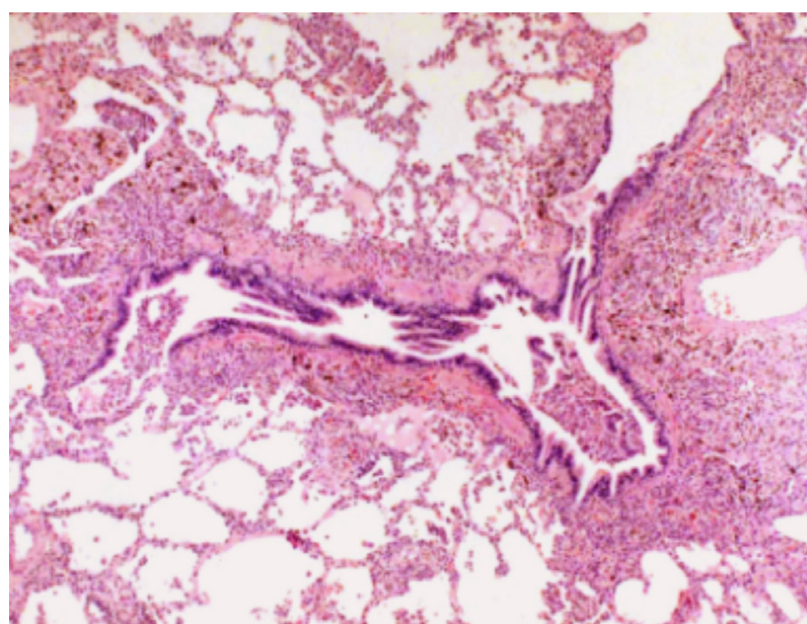

Figure 3. Infiltration of small to large lymphocytes into the alveolar septum and peribronchial inter-stitium $(\mathrm{H} \& \mathrm{E}, \times 400)$.

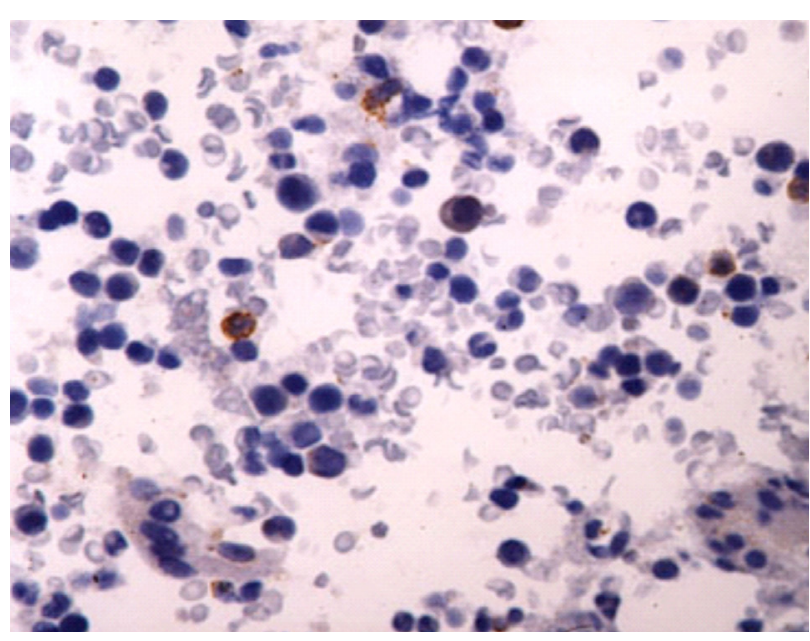

Figure 4. Numerous CD3-positive T lymphocytes (immunohistochemistry, $\times 400$ ).

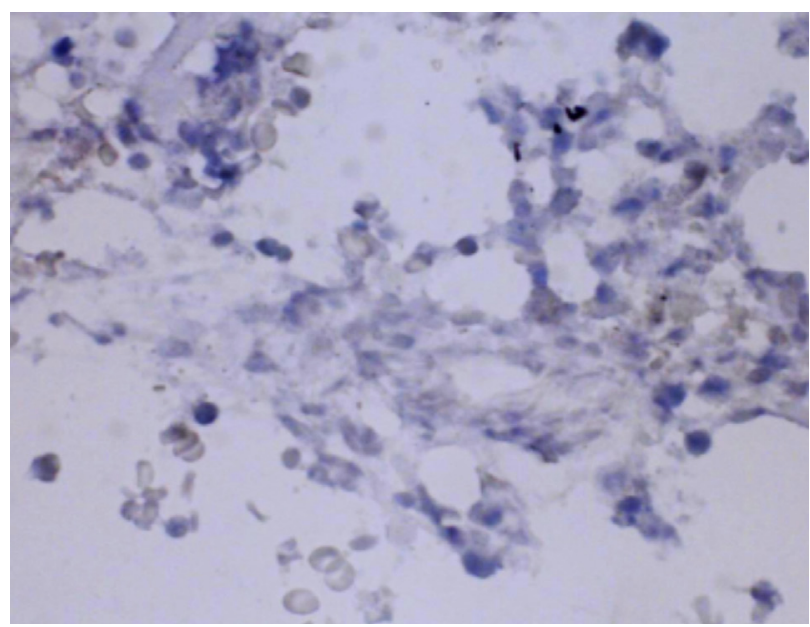

Figure 5. Intracellular Epstein-Barr virus (EBV) DNA detected in lymphoid cells (EBV-encoded RNA in situ hybridization, $x$ 400).
Twenty-six days after admission, her symptoms and signs had resolved spontaneously and she was discharged from the hospital. She was followed for 18 months. Six months after discharge, her EBV-DNA copy number had increased to 6,936 copies $/ 5 \mu \mathrm{L}$, but she remained well clinically over the entire follow-up period.

\section{DISCUSSION}

In 1978, Virelizier et al. [4] reported the case of a young female patient with very high IgG antibody titers to VCA and EA, accompanied by EBNA-positive cells in the blood and lymph nodes. Additional reports described a similar illness in another young female in 1984 [5] and in seven Japanese patients in 1986 [6]. In 1988, Straus [7] proposed diagnostic criteria for this syndrome, which he called severe and chronic EBV infection. In 2005, Okano et al. [3] has subsequently proposed another set of diagnostic criteria for this condition, which he called CAEBV infection syndrome, based on a review of the literature and their further clinical experience.

The proposed diagnostic criteria included the following: 1) persistent or recurrent infectious mononucleosis-like symptoms; 2) an unusual pattern of EBV antibodies with elevated anti-VCA and anti-EA, or detection of the EBV genome in affected tissues including the peripheral blood; and 3) chronic illness that cannot be explained by any other known disease processes at the time of diagnosis [3]. Our case met all of these criteria.

In our case, both immunohistochemistry and in situ hybridization with an EBER probe enabled us to detect numerous $\mathrm{CD}_{3}{ }^{+} \mathrm{T}$ lymphocytes that were infiltrating the lungs and contained the EBV genome in their nuclei. In CAEBV, unlike classic infectious mononucleosis, $\mathrm{T}$ lymphocytes or NK cells contain the EBV genome rather than $B$ cells. In addition, most patients with CAEBV have defective EBV-specific cytotoxic T cells, NK cells, and lymphokine-activated killer activity, which involve the defective production of several cytokines, such as interferon gamma and interleukin-1. The etiology of CAEBV remains unclear; however, it is possible that defective EBV replication in T/ NK cells and aberrant EBV-infected T/NK cell proliferation are major factors in the development of CAEBV $[2,6]$.

The major clinical features of CAEBV are fever, hepatosplenomegaly, liver dysfunction, pancytopenia, lymph- 
adenopathy, hypersensitivity to mosquito bites, skin rash, and uveitis [3]. In addition, CAEBV often results in lifethreatening complications, such as hemophagocytic syndrome, disseminated intravascular coagulopathy, hepatic failure, coronary artery aneurysm, central nervous system involvement, myocarditis, and interstitial pneumonitis [2].

The literature describes three pulmonary manifestations associated with EBV infection: hilar/mediastinal lymphadenopathy, pleural effusion, and interstitial pneumonitis. Few reports describe pulmonary parenchymal involvement as a complication of acute or chronic active EBV infection in immunocompetent patients [8,9]. A report on two children with CAEBV stated that the histopathology of their lung tissues showed interstitial infiltration of mature lymphocytes, which spread into the interalveolar septa, and similar to our case, EBV-positive T lymphocytes were detected throughout the alveolar septae and vascular lumens [9].

We report an immunocompetent adult who had a clinical syndrome of CAEBV and interstitial pneumonitis and pleural effusion associated with the infiltration of EBVinfected $\mathrm{T}$ lymphocytes into the lungs. This is the first known case of CAEBV-associated interstitial pneumonitis in Korea.

\section{Conflict of interest}

No potential conflict of interest relevant to this article was reported.

\section{REFERENCES}

1. Cohen JI. Epstein-Barr virus infection. N Engl J Med 2000; 343:481-492.

2. Kimura H. Pathogenesis of chronic active Epstein-Barr virus infection: is this an infectious disease, lymphoproliferative disorder, or immunodeficiency? Rev Med Virol 2006;16:251-261.

3. Okano M, Kawa K, Kimura H, et al. Proposed guidelines for diagnosing chronic active Epstein-Barr virus infection. Am J Hematol 2005;80:64-69.

4. Virelizier JL, Lenoir G, Griscelli C. Persistent Epstein-Barr virus infection in a child with hypergammaglobulinaemia and immunoblastic proliferation associated with a selective defect in immune interferon secretion. Lancet 1978;2:231-234.

5. Joncas JH, Ghibu F, Blagdon M, Montplaisir S, Stefanescu I, Menezes J. A familial syndrome of susceptibility to chronic active Epstein-Barr virus infection. Can Med Assoc J 1984;130:280284 .

6. Okano M, Sakiyama Y, Matsumoto S, Mizuno F, Osato T. Unusual lymphoproliferation associated with chronic active Epstein-Barr virus infection. AIDS Res 1986;2 Suppl 1:S121-S123.

7. Straus SE. The chronic mononucleosis syndrome. J Infect Dis 1988;157:405-412.

8. Ankermann T, Claviez A, Wagner HJ, Krams M, Riedel F. Chronic interstitial lung disease with lung fibrosis in a girl: uncommon sequelae of Epstein-Barr virus infection. Pediatr Pulmonol 2003;35:234-238.

9. Schooley RT, Carey RW, Miller G, et al. Chronic Epstein-Barr virus infection associated with fever and interstitial pneumonitis: clinical and serologic features and response to antiviral chemotherapy. Ann Intern Med 1986;104:636-643. 